\title{
Dimensión socioambiental en los contenidos del currículo del nivel medio superior de la Universidad Autónoma de Guerrero
}

\author{
Socio-Environmental Dimension in the Contents of the Curriculum of the \\ Universidad Autónoma de Guerrero's High School
}

\section{Dimensão socioambiental no conteúdo do currículo do nível médio superior da Universidade Autônoma de Guerrero}

\author{
Herlinda Gervacio Jiménez \\ Universidad Autónoma de Guerrero, Guerrero, México \\ lindagj09@gmail.com \\ https://orcid.org/0000-0003-3037-9528
}

Benjamín Castillo Elías

Universidad Autónoma de Guerrero, Instituto de Investigación Científica Humanístico Social,

Guerrero, México

bcastilloe@hotmail.com

https://orcid.org/0000-0003-1487-5353

\section{Resumen}

La presente investigación atiende a los requerimientos del nuevo modelo educativo. Se trata de un análisis de los contenidos del currículo del nivel medio superior de la Universidad Autónoma de Guerrero (UAGro), ubicada en Acapulco, Gro., México. Las cualidades o criterios que se utilizaron para el análisis de las variables fueron la vinculación con la educación ambiental, justificación, investigación sobre cuestiones ambientales, contenido científico ambiental, así como terminología específica ambiental. Los resultados obtenidos muestran que son cuatro las unidades de aprendizaje, a saber: Química I, II, III; Biología I, II; Geografía, y Ecología, las que presentan cuestiones ambientales con mayor profundidad y cuyos contenidos están relacionados con la sustentabilidad. La asignatura con más contenidos ambientales y educación ambiental es Ecología, 


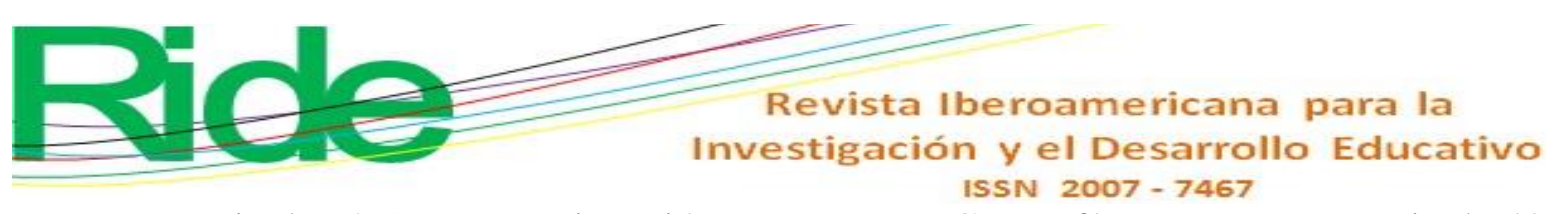

con un porcentaje de $62.5 \%$; a continuación se encuentra Geografía, con un porcentaje de 40.62 $\%$; seguida de la asignatura de Biología con un porcentaje de $12.5 \%$ de vinculación con temas ambientales y educación ambiental; por último, la asignatura con menor vinculación ambiental es Química, con tan solo $3.12 \%$. De acuerdo con lo anterior, los programas del nivel medio superior deben ser revisados y rediseñados de tal manera que atiendan de forma integral las necesidades educativas actuales.

Palabras clave: análisis curricular, contenidos curriculares, educación media superior, modelo educativo, transversalización.

\section{Abstract}

This research addresses the requirements of the new educational model. It is an analysis of the contents of the curriculum of the higher level of the Universidad Autónoma de Guerrero (UAGro), located in Acapulco, Gro., Mexico. The qualities or criteria that were used for the analysis of the variables were the link with environmental education, justification, research on environmental issues, environmental scientific content, as well as specific environmental terminology. The results obtained showed that there are four learning units (Chemistry I, II, III; Biology I, II; Geography, and Ecology) that present environmental issues with greater depth and related in their contents with sustainability. The subject with the least environmental connection to the contents of the subjects is Chemistry, with only 3.12\%; followed by the subject of Biology, with a percentage of $12.5 \%$ linked to environmental issues and environmental education; Geography with a percentage of $40.62 \%$, and finally the subject with the most environmental content and environmental education is Ecology, with a percentage of $62.5 \%$. According to the above-mentioned analysis, the programs of the upper secondary level must be revised and redesigned in such a way that they address in a comprehensive manner the current educational needs.

Keywords: curricular analysis, curricular contents, high school education, educational model, mainstreaming. 


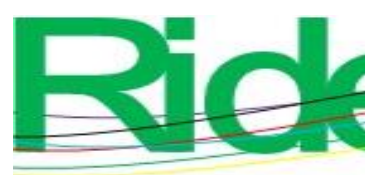

Revista Iberoamericana para la Investigación y el Desarrollo Educativo

ISSN 2007 - 7467

Siguiendo la actual reforma educativa, el currículo escolar debe de estar enfocado en el desarrollo de habilidades y destrezas que permitan tener una mejor calidad de vida personal, profesional y social y no simplemente en ser un medio para adquirir información (Delors, 1996; Pozo, 1997; Millar y Osborne, 1998; Burden, 2005; Chamizo, 2013).

Este interés se ha evidenciado en varios estudios y propuestas didácticas dirigidas a la mejora de la enseñanza-aprendizaje que incluyen desde el reconocimiento de las ideas previas de los estudiantes, las estrategias para lograr el cambio conceptual, el diseño de unidades didácticas diversas, el uso de progresiones de aprendizaje (Talanquer, 2013), la indagación y la resolución de problemas (Posner, 2005; Chamizo, 2013) hasta la incorporación de las tecnologías de la información y la comunicación (TIC). Asimismo, se han hecho propuestas que hablan de la necesidad de incluir no solo temas conceptuales, sino también aspectos relacionados con la naturaleza de la ciencia.

Estudios realizados sobre el tema curricular — como los de Lledó y Cano (1994), Novo (1996), Nieto (1999), Arbat y Geli (2002), Posner (2005), Serrano y Serrano (2007), Coll (2013), Watson, Lozano, Noyes y Rodgers (2013), Abero, Berardi, Capocasale, García y Rojas (2015), Yus (2015) y Colombo y Carvalho (2017) — dan cuenta de un profundo interés y grado de reflexión. En sus estudios sobresale la importancia de la integración e incorporación de contenidos sobre sustentabilidad ambiental en los planes de estudio.

Dentro del marco curricular común (MCC) de la educación media superior se establecen los objetivos generales y particulares para el desarrollo de competencias fundamentales que permitan a los egresados desarrollarse en todas sus dimensiones. Esto incluye las herramientas necesarias para la vida familiar y ciudadana, el aprendizaje socioemocional, el aprendizaje permanente y el ejercicio de la autonomía personal (Secretaría de Educación Pública [SEP], 2016b).

\section{Planteamiento del problema}

Los contenidos del actual currículo del nivel medio superior no contemplan las problemáticas ambientales actuales. De igual manera, no reflejan temas transversales para promover el equilibrio social-ecológico-ambiental que tanta falta hace a la sociedad y a la propia institución educativa desde la educación formal. 


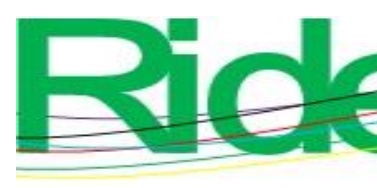

\section{Revista Iberoamericana para la Investigación y el Desarrollo Educativo ISSN 2007 - 7467}

\section{Justificación}

En marzo de 2016, se presentó un planteamiento para la actualización del modelo educativo (Secretaría de Educación Pública [SEP], 2016a). Dicho planteamiento estaba constituido por los siguientes tres documentos:

1. Carta sobre los Fines de la Educación para el siglo XXI: Expone de manera breve qué mexicanas y mexicanos se busca formar con el nuevo modelo educativo.

2. El Modelo Educativo 2016: Explica, en cinco grandes ejes, el modelo que se deriva de la reforma educativa, es decir, la forma en que se propone articular los componentes del sistema para lograr el máximo logro de aprendizaje de niños y jóvenes.

3. Propuesta Curricular para la Educación Obligatoria 2016: Un planteamiento curricular para la educación básica y la media superior, y abarca tanto la estructura de los contenidos educativos como los principios pedagógicos que la sustentan.

\section{Nuevo perfil de egreso de la educación media superior}

El perfil de egreso de la educación media superior resultó fortalecido a partir del MCC. Ahora incluye habilidades socioemocionales y competencias del siglo XXI para que los jóvenes puedan enfrentar con éxito los desafíos de la sociedad actual (Secretaría de Educación Pública [SEP], 2016b).

Dentro del nuevo perfil de egreso del estudiante del nivel medio superior se establece el ámbito "Cuidado del medio ambiente". A partir de éste, el estudiante "comprende la importancia de la sustentabilidad y asume una actitud proactiva para encontrar soluciones sustentables"; "Piensa globalmente y actúa localmente"; "Valora el impacto social y ambiental de las innovaciones y avances científicos” (Secretaría de Educación Pública [SEP], 2016a).

La visión que se tiene en la UAGro para el 2020 es que exista una transformación curricular: un plan de estudios flexible, evaluado y corregido sistemáticamente por las instancias colegiadas de la institución; programas renovados, de acuerdo con los avances de la ciencia y las necesidades sociales, con contenidos actualizados, respaldados por libros de texto en cada asignatura, que serán producto de un trabajo colectivo de las academias (Universidad Autónoma de Guerrero [UAGro], 2017).

Ahora bien, el concepto educación ambiental aparece en contextos de desarrollo cultural y tecnológico; surge de la toma de conciencia de los efectos que el desarrollo de las comunidades humanas produce en el medio natural, y surge también de la toma de conciencia de los alcances, a 


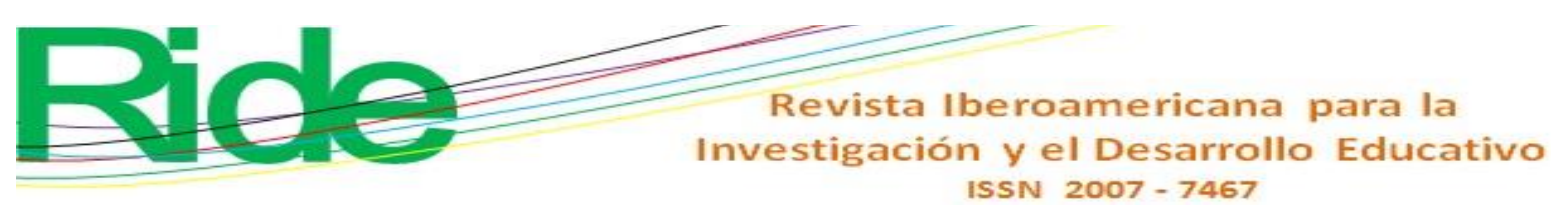

corto y largo plazo, de dichos efectos en el equilibrio de la biósfera, que es la sustentadora de la supervivencia física de los humanos.

La expresión educación ambiental aparece por primera vez en su versión en inglés (environmental education) en 1948 durante una reunión de la organización Unión Mundial para la Conservación de la Naturaleza (UICN). Se trata de un área de conocimiento que desarrolla un cuerpo conceptual abarcando a múltiples disciplinas no solo referentes al mundo científico, sino también al social, geográfico, económico y político, teniendo que evolucionar continuamente y de modo acelerado. En dicha evolución han incorporado contenidos a medida que han aparecido en el medio físico en las últimas décadas (agujeros en la capa de ozono, cambio climático, dosis elevadas de contaminantes). Además, ha tenido que desarrollar metodologías didácticas que posibiliten la adquisición de conductas y valores inductores de una actuación diferente ante los problemas ambientales.

En los años 80, la Organización de las Naciones Unidas para la Educación, la Ciencia y la Cultura (Unesco) promovió a nivel mundial la incorporación de la educación ambiental en los planes y programas de estudios, al igual que impulsar la renovación de programas nacionales de educación escolar y fortalecer redes internacionales, nacionales y regionales con la finalidad de intercambiar experiencias relacionadas con innovaciones pedagógicas en los campos de la educación y la formación sobre medio ambiente.

\section{Objetivo}

Analizar los contenidos del currículo del nivel medio superior de la UAGro y la relación de las asignaturas con la sustentabilidad y temas ambientales.

\section{Metodología}

La presente investigación se desarrolló en dos etapas. La primera etapa fue realizar una exploración diagnóstica del plan de estudios del nivel medio superior con la finalidad de indagar el nivel de impacto social teniendo como referente el MCC, el cual establece las competencias que requieren los jóvenes para enfrentarse a las nuevas condiciones del siglo XXI. Estas competencias fueron la base del análisis. Un análisis, cabe señalar, esencialmente cualitativo que dio paso a métodos teóricos: analítico-sintético, que permitió el análisis de las fuentes teóricas y los contenidos de la malla curricular de las asignaturas que se llevan en este nivel; inductivo-deductivo, 


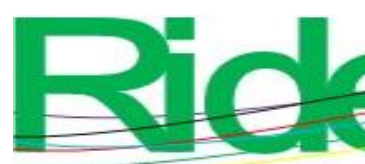

\section{Revista Iberoamericana para la Investigación y el Desarrollo Educativo ISSN 2007 - 7467}

gracias al cual se obtuvieron reflexiones acerca de las competencias y el perfil de egreso que debe poseer un egresado del nivel superior.

Para la revisión y análisis del plan y programas de estudios se utilizó el diseño denominado análisis de contenido, una técnica para estudiar y analizar la comunicación (programas televisivos o radiofónicos, artículos de prensa, reglamentos, programa de estudios) y, a partir de ahí, hacer inferencias válidas y confiables de datos con respecto a su contexto (Hernández, Fernández y Baptista, 2014).

La población o universo estudiado corresponde al conjunto de todos los casos que concuerdan con una serie de especificaciones (Hernández, Fernández y Baptista, 2014). En este caso, es el plan de estudios y el programa de estudio de las asignaturas de Ecología, Biología, Geografía, y Química del nivel medio superior.

Las unidades de análisis corresponden a los segmentos o partes del contenido de los mensajes que son caracterizados para ubicarlos en categorías (Hernández, Fernández y Baptista, 2014). Para esta investigación, las unidades de análisis estudiadas fueron la claridad y precisión de la información que contemplan los contenidos programáticos de las asignaturas.

La segunda etapa consistió en establecer el nivel de vinculación entre las competencias establecidas por el MCC y los contenidos de corte ambiental de las asignaturas mediante los siguientes niveles de vinculación: alta, media, baja, y muy baja (ver tabla 1). Así, los porcentajes correspondieron a la siguiente escala: alta vinculación de $75 \%$ a $100 \%$, media de $50 \%$ a $75 \%$, baja de $25 \%$ a $50 \%$ y muy baja de $0 \%$ a $25 \%$.

Tabla 1. Nivel de vinculación entre la educación ambiental y los contenidos de asignaturas

\begin{tabular}{|c|c|}
\hline $\begin{array}{c}\text { Porcentaje de vinculación entre los } \\
\text { contenidos de la asignatura y la educación } \\
\text { ambiental }(\%)\end{array}$ & Nivel de vinculación \\
\hline 0 a 25 & Muy Baja \\
\hline 25 a 50 & Baja \\
\hline 50 a 75 & Media \\
\hline 75 a 100 & Alta \\
\hline
\end{tabular}

Fuente: Elaboración propia

Las cualidades o criterios que se utilizaron para el análisis de las variables fueron los siguientes: 


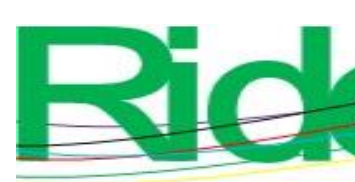

- Vinculación con la educación ambiental.

\section{Revista Iberoamericana para la Investigación y el Desarrollo Educativo ISSN 2007 - 7467}

- Justificación.

- Investigación sobre cuestiones ambientales.

- Contenido científico ambientales.

- Terminología específica ambiental.

\section{Resultados}

Con base en el análisis de los programas de estudio, se observó que existe cierta problemática de pertinencia y relevancia, así como dispersión y rigidez, en cuanto a los contenidos del programa de estudio (ver figura 1).

Figura 1. Pertinencia y relevancia de contenidos del programa de estudio

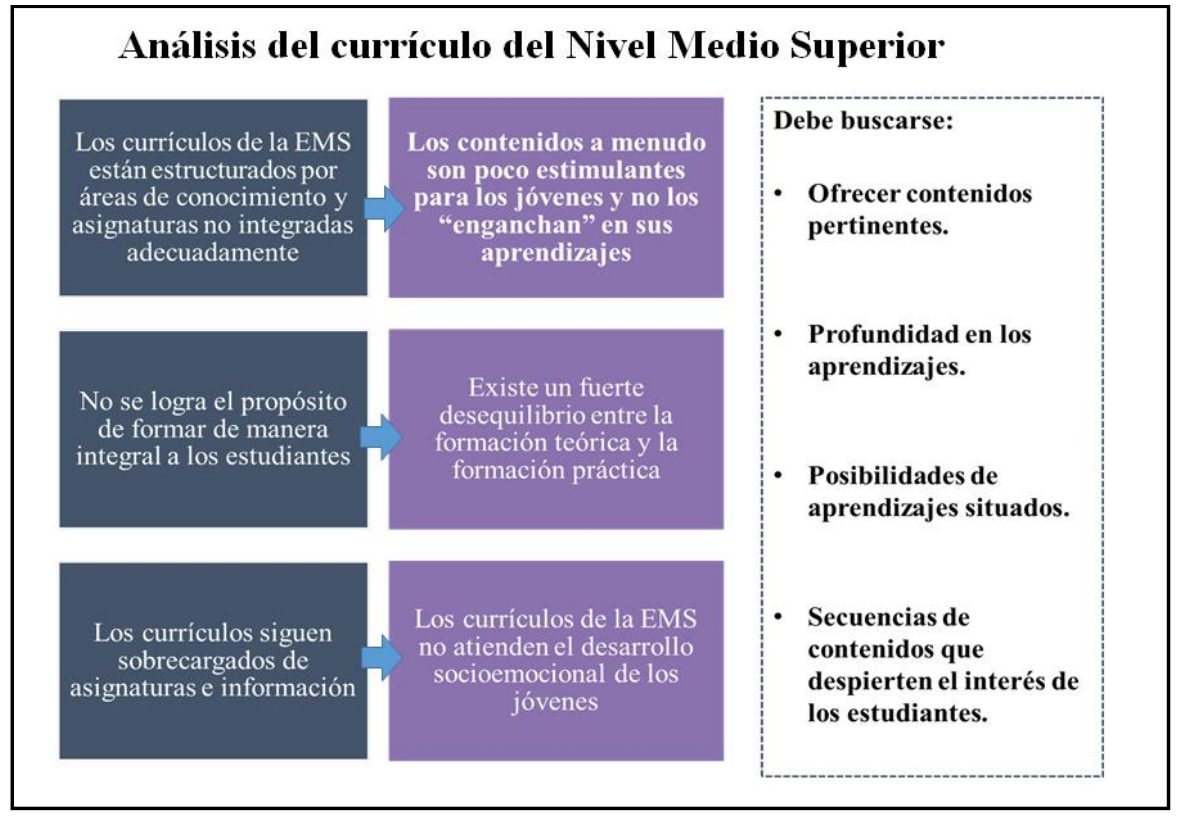

Fuente: Elaboración propia con base en (Secretaría de Educación Pública [SEP], 2016b).

El análisis muestra que los contenidos se centran en los estudiantes y en sus procesos de aprendizaje. Asimismo, plantean diferentes estrategias para generar ambientes de aprendizaje adecuados: se promueve el trabajo colaborativo con la finalidad de construir aprendizajes poniendo especial énfasis en el desarrollo de competencias; el logro de los aprendizajes esperados, así como la propuesta de poner en marcha el uso de materiales educativos que favorezcan los aprendizajes y una evaluación continua; la inclusión para atender la diversidad; están incorporados temas de interés sociales, al igual que la inclusión y participación activa de los padres de familia en el 


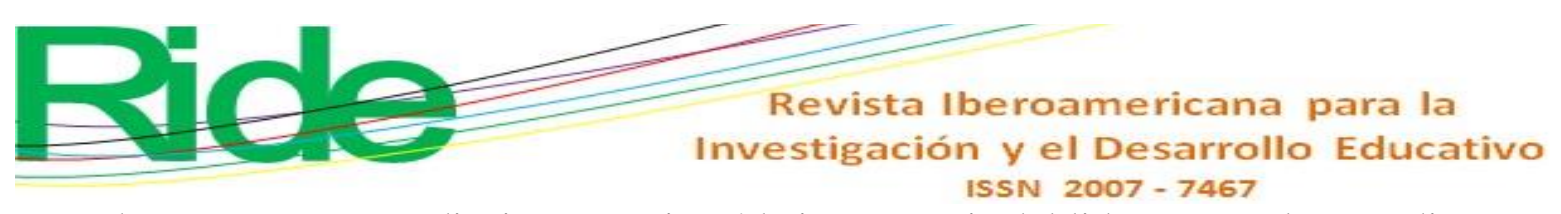

proceso de enseñanza-aprendizaje; se reorientó la importancia del liderazgo en los estudiantes y se puso especial énfasis a la tutoría y asesoría académica.

\section{La ambientalización del currículum}

Los resultados del análisis de la relación de las asignaturas del plan de estudios del nivel medio superior con la ambientalización, temas de corte ambiental e impacto en la formación del estudiante, se presentan de manera porcentual en la tabla 2.

Tabla 2. Concentrado de resultados por asignatura: Relación de diferentes temáticas con la educación ambiental.

\begin{tabular}{|c|c|c|c|c|c|c|c|c|c|c|c|}
\hline $\begin{array}{l}\text { dica el peso (\%) que } \\
\text { nbiental, así como el }\end{array}$ & act & & $\begin{array}{l}\text { as } \\
\text { do }\end{array}$ & fat & ip & $\begin{array}{l}\text { estudio en relación } \\
\text { ttaria estudiar cuestio }\end{array}$ & ar & $\begin{array}{l}\text { ntal } \\
\text { das }\end{array}$ & n, & nas & or \\
\hline Asignatura & $\begin{array}{l}\text { 응 } \\
\text { 롤 }\end{array}$ & 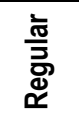 & ষ & 忐 & $\frac{\pi}{\frac{\pi}{2}}$ & Asignatura & $\frac{\text { 을 }}{\text { 를 }}$ & 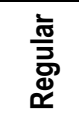 & ¿̊ & : & $\frac{\pi}{\frac{\pi}{0}}$ \\
\hline $\begin{array}{l}\text { Desarrollo biológico y } \\
\text { adolescente }\end{array}$ & $15 \%$ & $28 \%$ & $23 \%$ & $20 \%$ & $14 \%$ & Historia Universal & $11 \%$ & $19 \%$ & $19 \%$ & $22 \%$ & $29 \%$ \\
\hline Etimologías & $6 \%$ & $12 \%$ & $17 \%$ & $32 \%$ & $33 \%$ & Química & $13 \%$ & $24 \%$ & $25 \%$ & $22 \%$ & $16 \%$ \\
\hline Matemáticas & $8 \%$ & $14 \%$ & $15 \%$ & $22 \%$ & $41 \%$ & Economía & $12 \%$ & $16 \%$ & $20 \%$ & $23 \%$ & $29 \%$ \\
\hline $\begin{array}{l}\text { Orientación } \\
\text { Psicopedagógica }\end{array}$ & $15 \%$ & $20 \%$ & $19 \%$ & $22 \%$ & $24 \%$ & Física & $12 \%$ & $19 \%$ & $20 \%$ & $22 \%$ & $27 \%$ \\
\hline Taller de Arte & $12 \%$ & $18 \%$ & $20 \%$ & $24 \%$ & $26 \%$ & Geografía & $20 \%$ & $25 \%$ & $21 \%$ & $16 \%$ & $18 \%$ \\
\hline Educación Física & $19 \%$ & $16 \%$ & $15 \%$ & $22 \%$ & $27 \%$ & Estadística & $15 \%$ & $16 \%$ & $17 \%$ & $21 \%$ & $31 \%$ \\
\hline Inglés & $8 \%$ & $14 \%$ & $15 \%$ & $24 \%$ & $40 \%$ & Historia de México & $12 \%$ & $18 \%$ & $19 \%$ & $20 \%$ & $31 \%$ \\
\hline Psicología & $13 \%$ & $18 \%$ & $22 \%$ & $22 \%$ & $25 \%$ & $\begin{array}{l}\text { Metodología y técnicas de } \\
\text { investigación }\end{array}$ & $14 \%$ & $17 \%$ & $20 \%$ & $21 \%$ & $28 \%$ \\
\hline $\begin{array}{l}\text { Taller de lectura y } \\
\text { redacción }\end{array}$ & $9 \%$ & $16 \%$ & $18 \%$ & $23 \%$ & $34 \%$ & Taller de Cómputo & $13 \%$ & $14 \%$ & $13 \%$ & $19 \%$ & $41 \%$ \\
\hline Biología & $31 \%$ & $33 \%$ & $19 \%$ & $10 \%$ & $7 \%$ & Derechos Humanos & $13 \%$ & $19 \%$ & $18 \%$ & $19 \%$ & $31 \%$ \\
\hline Filosofía & $9 \%$ & $19 \%$ & $21 \%$ & $26 \%$ & $25 \%$ & Ecología & $40 \%$ & $25 \%$ & $18 \%$ & $9 \%$ & $8 \%$ \\
\hline Literatura & $10 \%$ & $17 \%$ & $19 \%$ & $23 \%$ & $31 \%$ & Seminario de & $1 \%$ & 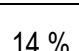 & $\%$ & $1 \%$ & \\
\hline Sociología & $11 \%$ & $19 \%$ & $20 \%$ & $26 \%$ & $24 \%$ & interdisciplinario & $10 \%$ & $14 \%$ & $13 \%$ & $21 \%$ & $2 \%$ \\
\hline
\end{tabular}

Fuente: Elaboración propia

Los resultados posicionan a la asignatura de Ecología con una ambientalización regular aun cuando se observó la falta de contenidos temáticos en relación con problemáticas ambiental y la educación ambiental, así como en la organización y enfoque de estos. Se encontraron algunos temas de corte ambiental que se abordan de manera aislada, además de algunos contenidos sobre el tópico en cuestión; en la última unidad contiene temas de educación ambiental.

En el caso de Química no se encontraron contenidos relacionados con la educación ambiental o la problemática ambiental. Dentro de esta asignatura los temas están al margen de los problemas concretos del ambiente vividos por los estudiantes. Con respecto a la asignatura de 


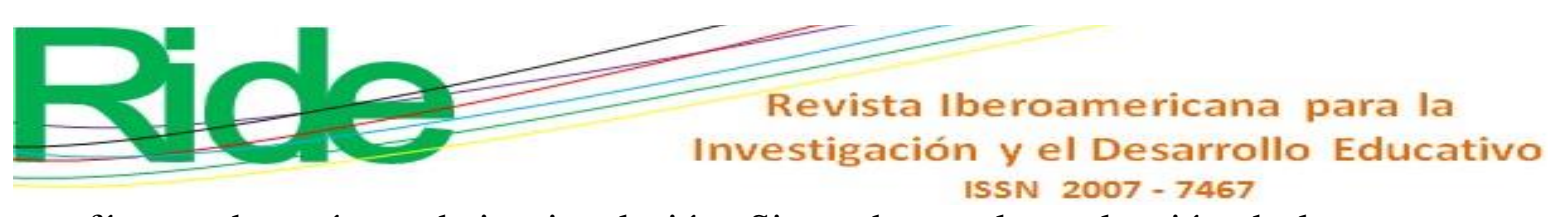

Geografía, se observó una baja vinculación. Sin embargo, la evaluación de los programas de estudio permitió ver que esta materia podría desempeñar un papel fundamental en el desarrollo de la educación ambiental. A través del estudio de los lugares que conforman los procesos físicos y humanos y la gente que vive en ellos, la geografía física estudia el marco de las actividades humanas, las influencias recíprocas del hombre y de su medio; mientras que la geografía humana se propone estudiar el espacio social, así como la utilización y ordenación del espacio geográfico, con miras a solucionar las necesidades de los hombres. Por lo tanto, la geografía ayudaría al estudiante a tener un mayor contacto con el mundo cercano. Los contenidos de esta asignatura tienen área de oportunidad, como el estudio de la calidad del entorno en una localidad, donde se propone que los estudiantes expresen sus gustos y disgustos sobre el ambiente estudiado, aprendan cómo el entorno es cambiante, opinen sobre cómo la calidad del ambiente debiera ser conservada o mejorada. La propuesta de la asignatura es que el estudiante debe ser llamado a reflexionar acerca de "las soluciones ambientales que dan a los problemas de la localidad estudiada, a la vez que reflexionarían sobre cómo la gente afecta al ambiente" (Serrano y Serrano, 2007).

El programa de Historia considera algunos temas objetivos de la educación ambiental, tal y como la estructuración de la noción de espacio, el paso del estudio del medio local al del planeta y de la noción de tiempo, de la región, del hombre y el medio que lo rodea, de las grandes aglomeraciones urbanas, de una región industrial; son temas a partir de los cuales pueden ser estudiados los principales problemas del ambiente. De acuerdo con Esteban (2001), los estudiantes adquieren ciertas actitudes que les permitan situarse en un mundo complejo, comprender los problemas planteados, ejercer sus derechos y respetar sus deberes como futuros ciudadanos.

Una vez realizado el análisis de los programas, las asignaturas de Química I, II, III, Biología I, II, Geografía y Ecología fueron las que presentaron mayor profundidad en sus contenidos ambientales.

\section{Análisis del programa de Química}

Se identificó que las asignaturas de Química I, II, III tienen el índice más bajo de ambientalización, con un porcentaje de $3.12 \%$ (véase tabla 3). 


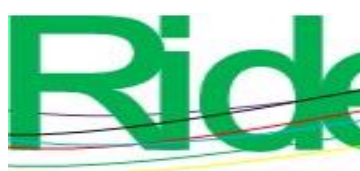

\section{Revista Iberoamericana para la Investigación y el Desarrollo Educativo ISSN 2007 - 7467}

Tabla 3. Nivel de vinculación entre los contenidos de las asignaturas de Química I, II, III y la educación ambiental en cuanto a claridad y precisión

\begin{tabular}{|c|c|}
\hline Variables independientes & Indicadores \\
\hline $\begin{array}{l}\text { Tendencias de la educación ambiental en el nivel medio superior en la } \\
\text { materia de Química. }\end{array}$ & $\begin{array}{ll}> & \text { Vinculación } \\
> & \text { Justificación } \\
> & \text { Investigación } \\
> & \text { Contenido científico } \\
> & \text { Terminología específica }\end{array}$ \\
\hline Profundidad de los aprendizajes sobre el medio ambiente. & $25 \%$ \\
\hline Conocimientos sobre la crisis ambiental actual. & $0 \%$ \\
\hline Conocimientos sobre la dimensión social de la problemática ambiental. & $0 \%$ \\
\hline Análisis de cuestiones legales sobre el ambiente. & $0 \%$ \\
\hline Aplicación de los conocimientos sobre ambiente al contexto próximo. & $0 \%$ \\
\hline Estudio de la realidad ambiental a nivel mundial. & $0 \%$ \\
\hline Estudio de la problemática ambiental en México. & $0 \%$ \\
\hline Estudio de la realidad ambiental en Acapulco. & $0 \%$ \\
\hline $\begin{array}{l}\text { Porcentaje total de vinculación entre los contenidos de la Asignatura y la } \\
\text { educación ambiental }\end{array}$ & $3.12 \%$ \\
\hline
\end{tabular}

\section{Fuente: Elaboración propia}

La justificación para introducir la asignatura de Química I dentro del currículum es proponerla como una "cultura básica" que le permita al estudiante observar, comprender y explicar los fenómenos químicos que en todo momento y de manera incesante se producen en la naturaleza. En el caso de Química III, la justificación de la asignatura para ser incluida dentro del currículum es que se centra en el estudio de algunos componentes químicos de alimentos especialmente importantes por su papel nutritivo. A través de dicho acercamiento el alumno podrá apreciar que existen compuestos químicos imprescindibles para la estructura y funcionamiento de los organismos vivos y que los procesos de la vida tienen sus orígenes en una serie de reacciones químicas. También obtendrá conocimientos que le ayudarán a llevar una dieta balanceada.

Por lo que se observó, no existe un vínculo directo con la problemática ambiental actual, no existe una vinculación y aplicación práctica para resolver problemas reales con el ambiente.

Los conceptos son estrictamente técnicos, sin ninguna relación con el contexto próximo, no se habla de aplicar cuestiones ambientales con los conocimientos adquiridos respecto a elementos químicos, así como tampoco se abordan temas relacionados con el cuidado de los recursos naturales que se estudian en algunos apartados de las unidades respectivas. 


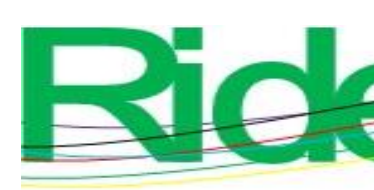

\section{Revista Iberoamericana para la Investigación y el Desarrollo Educativo ISSN $2007-7467$}

Existe otra asignatura de química de manera optativa, la cual es una introducción para los alumnos que se perfilen a estudios más profundos en cuestiones químicas.

\section{Análisis del programa de Biología I y II. Categoría: Claridad o precisión}

La biología, por tratarse de una ciencia que estudia a los seres vivos, contempla un enfoque más apegado a las cuestiones ambientales. Sin embargo, se observó que prácticamente el contenido temático de esta asignatura está dirigido hacia la apropiación de términos biológicos y uso del método experimental.

La justificación de la asignatura dentro del plan de estudios es que, con los contenidos programáticos de Biología I que se desarrollarán en el segundo semestre, se pretende que los estudiantes: $a$ ) se apropien de los conceptos biológicos fundamentales y que se familiaricen con el uso del método experimental como la forma más adecuada para la consecución de conocimientos significativos en esta disciplina científica; $b$ ) desarrollen una concepción científica sobre el origen de la vida y del universo, partiendo, para ello, del análisis y discusión de las distintas teorías que intentan explicar dichos fenómenos, y c) comprendan la estructura y las funciones de la célula para que, mediante los postulados de la teoría celular, puedan explicar la organización y complejidad de los seres vivos. Como se observa en la justificación, se contemplan aspectos estrictamente técnicos biológicos, por lo que está muy lejos de acercarse a lo que es la educación ambiental.

El resultado del análisis del programa muestra que en las variables "Información sobre el medio ambiente en sucesos pasados" y "Conocimientos sobre la crisis ambiental actual” solo se contemplan las cuestiones ambientales en $25 \%$; con lo que respecta a los demás indicadores su vinculación es prácticamente nula. Con un total, en cuanto a las tendencias de la asignatura de Biología con respecto a la educación ambientales, de $12.5 \%$.

A partir de lo anterior, se asevera que los contenidos de esta asignatura están más relacionados con las cuestiones del ambiente puro, sin involucrar la dimensión social, de qué manera afectan, es decir, sin considerar la problemática ambiental, y menos considerando la aplicación de los conocimientos adquiridos en esta asignatura en la vida cotidiana. Su aplicación va más enfocada a cuestiones técnicas de la biología, y en general son conocimientos que raramente se aplican para resolver la problemática ambiental a nivel nacional o local. En la tabla 4 se muestran los resultados de este análisis. 


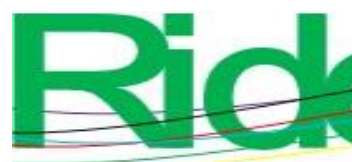

\section{Revista Iberoamericana para la Investigación y el Desarrollo Educativo ISSN 2007 - 7467}

La tendencia de la asignatura de Geografía en relación con la educación ambiental y los problemas ambientales es de $40.62 \%$.

Tabla 5. Nivel de vinculación entre los contenidos de la asignatura de Geografía con la educación ambiental en cuanto a claridad y precisión

\begin{tabular}{|c|c|}
\hline VARIABLES INDEPENDIENTES & INDICADORES \\
\hline $\begin{array}{l}\text { Tendencias de la educación ambiental en el nivel medio superior en la } \\
\text { materia de Geografía. }\end{array}$ & $\begin{array}{ll}> & \text { Vinculación } \\
> & \text { Justificación } \\
> & \text { Investigación } \\
> & \text { Contenido científico } \\
> & \text { Terminología } \\
& \text { específica }\end{array}$ \\
\hline Profundidad de los aprendizajes sobre el ambiente. & $75 \%$ \\
\hline Conocimientos sobre la crisis ambiental actual. & $25 \%$ \\
\hline Conocimientos sobre la dimensión social de la problemática ambiental. & $75 \%$ \\
\hline Análisis de cuestiones legales sobre el medio ambiente. & $50 \%$ \\
\hline Aplicación de los conocimientos sobre ambiente al contexto próximo. & $25 \%$ \\
\hline Estudio de la realidad ambiental a nivel mundial. & $25 \%$ \\
\hline Estudio de la problemática ambiental en México. & $25 \%$ \\
\hline Estudio de la realidad ambiental en Acapulco. & $25 \%$ \\
\hline $\begin{array}{l}\text { Porcentaje total de vinculación entre los contenidos de la asignatura y la } \\
\text { educación ambiental }\end{array}$ & $40.62 \%$ \\
\hline
\end{tabular}

Fuente: Elaboración propia

\section{Análisis del programa de Ecología. Categoría: Claridad o precisión}

En el programa se encuentra la asignatura como Principios de Ecología, la cual se imparte en el sexto semestre y es el único donde se presenta esta asignatura de los seis que comprende el plan de estudios.

Los resultados obtenidos muestran que es la materia con mayor contenido ambiental. De acuerdo con los indicadores, la tendencia hacia la educación ambiental es de $62.5 \%$ (ver tabla 6).

En lo que respecta a la variable "Información sobre el ambiente en sucesos pasados" arroja un nivel de vinculación de $50 \%$ en sus contenidos programáticos. La variable "Información sobre la dimensión ambiental social de la problemática ambiental”, por su parte, tiene un nivel de contenidos de $75 \%$, el mismo porcentaje que en la variable "Conocimientos sobre la dimensión 


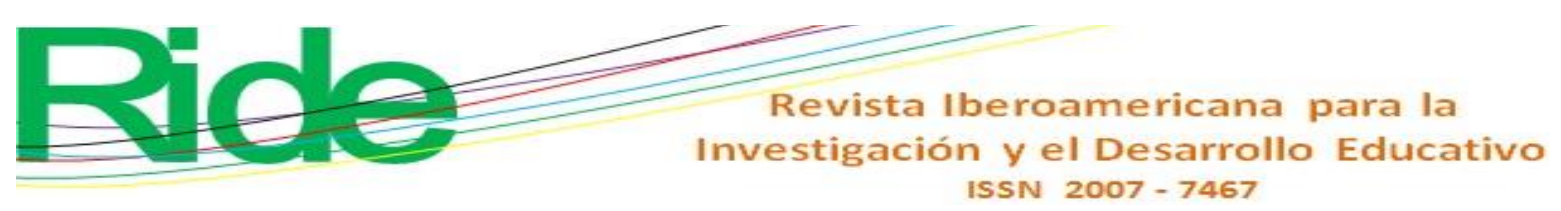

Flor, J. I. (2006). Hablemos del medio ambiente. Para conocer cómo funciona nuestro entorno y poder actuar. Madrid, España: Pearson-Alambra.

Foladori, G. y González, E. (2001). En pos de la Historia en educación ambiental. Tópicos en Educación Ambiental, 3(8), 28-43. Recuperado de http://www.anea.org.mx/Topicos/T\%208/Paginas\%2028\%20-\%2043.pdf.

González, E. (2003). Atisbando la construcción conceptual de la educación ambiental en México. En Berteley, M. (coord. ${ }^{a}$ ), Educación, Derechos Sociales y Equidad (Tomo I) (pp. 243272). Ciudad de México, México: Consejo Mexicano de Investigación Educativa. Recuperado de http://www.comie.org.mx/doc/portal/publicaciones/ec2002/ec2002 v03 t1.pdf.

Gough, N. (2000). Repensar el sujeto: (de)construyendo la acción humana en la investigación en educación ambiental. Tópicos en Educación Ambiental, 4(2) 49-60. Recuperado de http://www.anea.org.mx/wp-content/uploads/2015/02/Paginas-49-60-n04.pdf.

Hernández, R., Fernández, C. C. y Baptista, L. P. (2014). Metodología de la Investigación (6. ${ }^{\text {e ed.). }}$ México: McGraw-Hill.

Isaac-Márquez, R., Salavarría, O., Eastmond, A., Ayala, M., Arteaga, M., Isaac-Márquez, A., Sandoval, J. y Manzanero, L. (2011). Cultura ambiental en estudiantes de bachillerato. Estudio de caso de la educación ambiental en el nivel medio superior de Campeche. Revista Electrónica de Investigación Educativa, 13(2), 83-98. Recuperado de http://redie.uabc.mx/vol13no2/contenido-isaacmarquezetal.html.

Lledó, A. I. y Cano, M. I. (1994). Cambiar el entorno. Educación Primaria. Cuadernos de Pedagogía, (226), 22-25.

Millar, R. and Osborne, J. (eds.) (1998). Beyond 2000: Science education for the future. The report of a seminar series funded by the Nuffield Foundation. London, England: Kings's College. Recuperado de https://www.nuffieldfoundation.org/sites/default/files/Beyond\%202000.pdf.

Mrazek, R. (1996). Paradigmas alternativos de investigación en educación ambiental. (tesis de maestría). Universidad de Guadalajara, Jalisco.

Nieto, L. M. (1999). La perspectiva ambiental en los currículos profesionales ¿Una materia más? Revista Universitarios, 7(2), 1-14. Recuperado de http://ambiental.uaslp.mx/docs/LMNCAU-9905-EcolPlanEst.pdf. 


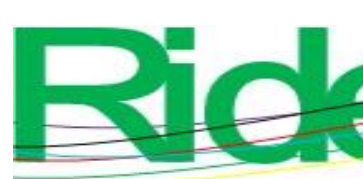

Revista Iberoamericana para la Investigación y el Desarrollo Educativo

ISSN 2007 - 7467

Novo, M. (1996). La educación ambiental: bases éticas, conceptuales y metodológicas (3. ${ }^{\mathrm{a}}$ ed.). Madrid, España: Editorial Universitas.

Peña, Y., Gámez, D., Carralero, Y., Sainz, A., Almaguer, L. y García, J. (2018). Procedimientos metodológicos para la implementación de la estrategia curricular Salud Pública y Educación Ambiental. EDUMECENTRO, 10(2), 6-20. Recuperado de http://www.revedumecentro.sld.cu/index.php/edumc/article/view/1055.

Posner, J. G. (2005). Análisis del currículo (3. ${ }^{\mathrm{a}}$ ed.). México: McGraw-Hill.

Pozo, J. I. (1997). La crisis de la educación científica, ¿volver a lo básico o volver al constructivismo? Alambique. Didáctica de las Ciencias Experimentales, (14), 91-104. Recuperado de http://hdl.handle.net/10486/668532.

Sauvé, L. (1999). Environmental Education Between Modernity and Postmodernity: Searching for an Integrating Educational Framework. Canadian Journal of Environmental Education, (4), 9-35.

Secretaría de Educación Pública [SEP]. (2016a). El Modelo educativo. El planteamiento pedagógico de la Reforma Educativa. México: Secretaría de Educación Pública. Recuperado

de https://www.sep.gob.mx/work/models/sep1/Resource/8007/1/images/modelo_educativo_ 2016.pdf.

Secretaría de Educación Pública [SEP]. (2016b). Propuesta curricular para la educación obligatoria. Ciudad de México, México: Secretaría de Educación Pública.. Recuperado de https://www.gob.mx/cms/uploads/docs/Propuesta-Curricular-baja.pdf.

Serrano, R. y Serrano, C. (2007). Ideología y educación ambiental: su incorporación en el currículo universitario, utopía y praxis. Educere, 11(36), 1-8. Recuperado de https://www.redalyc.org/html/356/35617701008/.

Talanquer, V. (2013). Progresiones de aprendizaje: promesa y potencial. Educación Química, 24(4), 362-364. Recuperado de https://core.ac.uk/download/pdf/82266229.pdf.

Universidad Autónoma de Guerrero [UAGro]. (2017). Plan Institucional de Desarrollo Estratégico Nivel Medio Superior. Guerrero, México: Universidad Autónoma de Guerrero. Dirección de Docencia. Departamento de Educación Media Superior. Recuperado de https://www.uagro.mx/conocenos/doc/PDI2017-2021.pdf.

Watson, M. K., Lozano, R., Noyes, C. and Rodgers, M. (2013). Assessing curricula contribution to sustainability more holistically: Experiences from the integration of curricula assessment 


\section{Revista Iberoamericana para la Investigación y el Desarrollo Educativo ISSN $2007-7467$}

and students' perceptions at the Georgia Institute of Technology. Journal of Cleaner
Production,
$(61)$
$106-116$.
Retrieved
from

https://www.sciencedirect.com/science/article/pii/S0959652613006136?via\%3Dihub.

Yus, R. (2015). Temas transversales: hacia una nueva escuela. Barcelona, España: Editorial Graó.

\begin{tabular}{|c|c|}
\hline Rol de Contribución & Autor (es) \\
\hline Conceptualización & Herlinda Gervacio Jiménez \\
\hline Metodología & Herlinda Gervacio Jiménez (igual), Benjamín Castillo Elías (igual) \\
\hline Software & Herlinda Gervacio Jiménez \\
\hline Validación & Herlinda Gervacio Jiménez (igual), Benjamín Castillo Elías (igual) \\
\hline Análisis Formal & Herlinda Gervacio Jiménez (igual), Benjamín Castillo Elías (igual) \\
\hline Investigación & Herlinda Gervacio Jiménez (igual), Benjamín Castillo Elías (igual) \\
\hline Recursos & Herlinda Gervacio Jiménez (igual), Benjamín Castillo Elías (igual) \\
\hline Curación de datos & Herlinda Gervacio Jiménez \\
\hline $\begin{array}{l}\text { Escritura - Preparación del borrador } \\
\text { original }\end{array}$ & Herlinda Gervacio Jiménez (igual), Benjamín Castillo Elías (igual) \\
\hline Escritura - Revisión y edición & Herlinda Gervacio Jiménez (igual), Benjamín Castillo Elías (igual) \\
\hline Visualización & Herlinda Gervacio Jiménez (igual), Benjamín Castillo Elías (igual) \\
\hline Supervisión & Herlinda Gervacio Jiménez (igual), Benjamín Castillo Elías (igual) \\
\hline Administración de Proyectos & Herlinda Gervacio Jiménez (igual), Benjamín Castillo Elías (igual) \\
\hline Adquisición de fondos & Herlinda Gervacio Jiménez (igual), Benjamín Castillo Elías (igual) \\
\hline
\end{tabular}

